\title{
Fundamental Function for the Lattice-based Random Walk Simulation in One Dimension
}

\author{
Joung-Hahn Yoon and Hyojoon Kim ${ }^{\dagger}$,* \\ Department of Mathematics, Dong-A University, Busan 604-714, Korea \\ ${ }_{\dagger}^{\dagger}$ Department of Chemistry, Dong-A University, Busan 604-714, Korea. ${ }^{*}$ E-mail: hkim@donga.ac.kr \\ Received March 3, 2011, Accepted July 18, 2011
}

Key Words : Diffusion-reaction, Monte carlo simulation, Random walk, Trap problem, Survival probability

Recently, diffusion-influenced reactions attract increasing attention. It is well-known that diffusion-influenced reactions can be described by lattice-based random walk Monte Carlo simulations. ${ }^{1-6}$ Since the continuum limit of the random walk model is closely related to the diffusion process, we can straightforwardly simulate general diffusionreaction systems with the lattice-based Monte Carlo simulation. Although the theories of the random walk on the lattice points have been regularly reported, ${ }^{1,7}$ the analytical results for diffusion-reaction systems on lattice points have been relatively rare.

In this Note, we find the fundamental distribution function of a random walker on the one-dimensional lattice with a reactive trap. This distribution function leads to a discrete version of the survival probability, which can be reduced to the well-known survival probability with the absorbing or Smoluchowski boundary condition. ${ }^{8}$

\section{Non-reactive Random Walk Model in One Dimension}

Firstly, we consider the random walk model without a reactive trap. Let $P_{N}\left(x, n ; x_{0}\right)$ be the non-reactive probability that the walker is observed at $x$ after $n$ steps with the starting position $x_{0}$ on the one-dimensional lattice. Suppose that jumps to the left and to the right occur with probability $p$ and $q=1-p$, respectively. After the first step, we have $P_{N}\left(x_{0}-\right.$ 1,$\left.1 ; x_{0}\right)=p$ and $P_{N}\left(x_{0}+1,1 ; x_{0}\right)=q$, and after two steps, $P_{N}\left(x_{0}-2,2 ; x_{0}\right)=p^{2}, P_{N}\left(x_{0}, 2 ; x_{0}\right)=2 p q$, and $P_{N}\left(x_{0}+2,2 ; x_{0}\right)=$ $q^{2}$, and so on. The probability that the walker moved $k$ times to the left and $n-k$ times to the right is given by the binomial distribution

$$
P_{N}\left(x, n ; x_{0}\right)={ }_{n} C_{k} p^{k} q^{n-k}
$$

By definition, $k=\left(x_{0}+n-x\right) / 2$ should be an integer satisfying $0 \leq k \leq n$ or $-n+x_{0} \leq x \leq n+x_{0}$, and ${ }_{n} C_{k}=$ $n ! /\{k !(n-k) !\}$ is not zero only when $k=n, n-1, \ldots, 0$ or $x=-n+x_{0}, \quad-n+x_{0}+2, \ldots, \quad n+x_{0}$. If the jump probabilities $p$ and $q$ are the same as $p=q=1 / 2$, the probability after $n$ steps is given by

$$
P_{N}\left(x, n ; x_{0}\right)=2^{-n} C_{\left(n-x+x_{0}\right) / 2} .
$$

One can see that the random walk model on the onedimensional lattice is closely related to Pascal's triangle, of which entries are binomial coefficients, illustrated in Figure 1(a). The probability after $n-1$ steps is related to the value in the $n$th row of Pascal's triangle. Note that $P_{N}$ is zero in every other column.

\section{Reactive Random Walk Model with a Perfect Trap}

Now, we consider the one-dimensional lattice-based random walk with a single static perfect trap. This reactive condition is related to the Smoluchowski or the absorbing boundary condition in diffusion-reaction systems. Suppose the trap is located at the origin and the starting position is $x_{0}=2$. Figure 1(b) illustrates such the discrete random walk. These entries are equal or less than those of the normal Pascal's triangle in Figure 1(a). While rightmost two entries are the same as those in the normal Pascal's triangle with $x_{0}=2$, the others are less than those in the normal Pascal's triangle since they are affected by the trap.

The difference between values in Figure 1(a) and in Figure 1(b) is shown in Figure 1(c). One can see that the rightmost two entries in each row are equal to 0 and all of the third

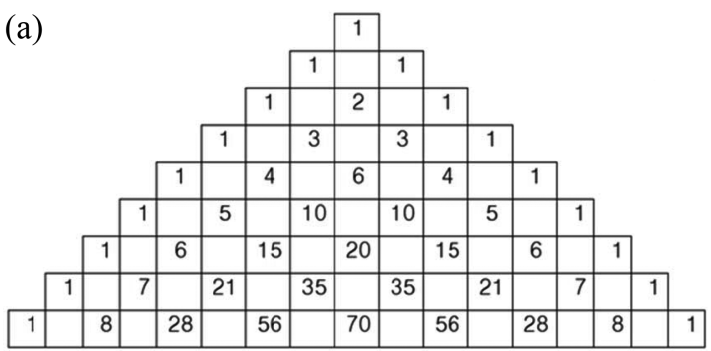

(b)

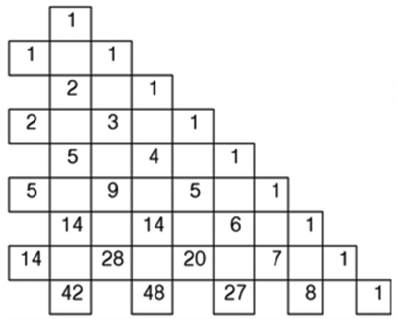

(c)

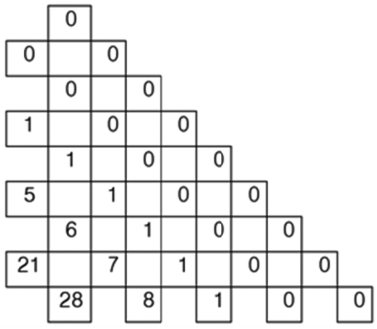

Figure 1. (a) Normal Pascal's triangle (b) Pascal's triangle with a reactive trap at origin. (c) The difference between values in (a) and (b). 
entries from the right are 1 . We find that this triangle is the same as the normal Pascal's triangle with the starting position $x_{0}=-2$. Let $P_{S M}\left(x, n ; x_{0}\right)$ be the probability that the walker is observed at $x$ after $n$ steps with the starting position $x_{0}$ for the Smoluchowski boundary condition at the origin. Without loss of generality, we can assume $x_{0}>0$. Then, we have

$$
\begin{aligned}
P_{S M}\left(x, n ; x_{0}\right) & =P_{N}\left(x, n ; x_{0}\right)-P_{N}\left(x, n ;-x_{0}\right) \\
& =2^{-n}\left\{{ }_{n} C_{\left(n-x+x_{0}\right) / 2}-{ }_{n} C_{\left(n-x-x_{0}\right) / 2}\right\}
\end{aligned}
$$

where $\left(n-x+x_{0}\right) / 2$ and $\left(n-x-x_{0}\right) / 2$ are the nonnegative integers. Since $P_{S M}\left(x, n ; x_{0}\right)$ is zero when $x<1$, the term ${ }_{n} C_{\left(n-x-x_{0}\right) / 2}$ contributes to $P_{S M}$ only when $n-x_{0}-1 \geq 0$. If $x_{0}$ is even, $P_{S M}\left(x, n ; x_{0}\right) \neq 0$ when $n-x$ is even. For odd $x_{0}, P_{S M}\left(x, n ; x_{0}\right) \neq 0$ for odd $n-x$. Note that $P_{S M}$ is zero at every other $x$ like $P_{N}$.

Let $S_{S M}\left(n ; x_{0}\right)$ be the survival probability after $n$ steps with the starting position $x_{0}$. We can obtain the survival probability $S_{S M}\left(n ; x_{0}\right)$ by summing $P_{S M}\left(x, n ; x_{0}\right)$ over $x$ as follows:

$$
\begin{aligned}
2^{n} S_{S M}\left(n ; x_{0}\right)= & 2^{n} \sum_{x=1}^{n+x_{0}} P_{S M}\left(x, n ; x_{0}\right) \\
= & { }_{n} C_{\left(n-1+x_{0}\right) / 2}-{ }_{n} C_{\left(n-1-x_{0}\right) / 2}+{ }_{n} C_{\left(n-3+x_{0}\right) / 2} \\
& -{ }_{n} C_{\left(n-3-x_{0}\right) / 2}+\cdots+{ }_{n} C_{0}, \\
= & \sum_{i=1}^{x_{0}}{ }_{n} C_{\left\lfloor\left(n-x_{0}-1\right) / 2\right\rfloor+i}
\end{aligned}
$$

where $\lfloor\cdots\rfloor$ is the greatest integer function or the floor function, in other words, $\lfloor x\rfloor$ is the greatest integer that is less or equal to $x$. Interestingly, when $x_{0}$ is even, $S_{S M}(n+1$; $\left.x_{0}\right)=S_{S M}\left(n ; x_{0}\right)$ for even $n$, and for odd $x_{0}, S_{S M}(n+1$; $\left.x_{0}\right)=S_{S M}\left(n ; x_{0}\right)$ for odd $n$ due to the floor function.

\section{Long Time Approximation}

As $n$ increases, we can utilize the following well-known de Moivre-Laplace theorem ${ }^{9}$

$$
\lim _{n \rightarrow \infty} C_{k} p^{k} q^{n-k}=\frac{1}{\sqrt{2 \pi n p q}} \exp \left(-\frac{(k-n p)^{2}}{2 n p q}\right),
$$

where $p+q=1, p>0$, and $q>0$. Thus, in the large $n$ limit or in the long time limit, the discrete binomial distribution $B(n, p)$ can be approximated by the normal distribution function $N(n p, n p q)$ with the mean value $n p$ and the variance $n p q$.

Since $p=q=1 / 2$ in our case, we obtain

$$
\lim _{n \rightarrow \infty} P_{N}\left(x, n ; x_{0}\right)=\sqrt{\frac{1}{2 \pi n}} \exp \left(-\frac{\left(x-x_{0}\right)^{2}}{2 n}\right)
$$

$$
\lim _{n \rightarrow \infty} P_{S M}\left(x, n ; x_{0}\right)=\sqrt{\frac{1}{2 \pi n}}\left\{\exp \left(-\frac{\left(x-x_{0}\right)^{2}}{2 n}\right)-\exp \left(-\frac{\left(x+x_{0}\right)^{2}}{2 n}\right)\right\} \text {. }
$$

It should be noted that we have multiplied the factor $1 / 2$ to obtain these equations since $P_{N}$ and $P_{S M}$ are zero at every other $x$ in their discrete versions. When $n=2 D t$, where $D$ is the diffusion constant and $t$ is the time, Eqs. (6) and (7) reduce to the well-known expressions ${ }^{8}$

$$
\begin{gathered}
P_{N}\left(x, n ; x_{0}\right)=\sqrt{\frac{1}{4 \pi D t}} \exp \left(-\frac{\left(x-x_{0}\right)^{2}}{4 D t}\right), \\
P_{S M}\left(x, n ; x_{0}\right)=\sqrt{\frac{1}{4 \pi D t}}\left\{\exp \left(-\frac{\left(x-x_{0}\right)^{2}}{4 D t}\right)-\exp \left(-\frac{\left(x+x_{0}\right)^{2}}{4 D t}\right)\right\},
\end{gathered}
$$

respectively.

Similarly, the survival probability $S_{S M}\left(n ; x_{0}\right)$ can be approximated by the following integral:

$$
\begin{aligned}
\lim _{n \rightarrow \infty} S_{S M}\left(n ; x_{0}\right) & =\int_{\left(n-x_{0}\right) / 2}^{\left(n+x_{0}\right) / 2} \sqrt{\frac{2}{\pi n}} \exp \left(-\frac{(2 x-n)^{2}}{2 n}\right) d x \\
& =\sqrt{\frac{1}{\pi}} \int_{-x_{0} / \sqrt{2 n}}^{x_{0} / \sqrt{2 n}} \exp \left(-u^{2}\right) d u, \\
& =\operatorname{erf}\left(\frac{x_{0}}{\sqrt{2 n}}\right)
\end{aligned}
$$

where $\operatorname{erf}(x)$ is the error function. The second equality comes from the substitution of $(2 x-n)^{2}=2 n u^{2}$. If $n=2 D t$, this equation is again the same as the well-known survival

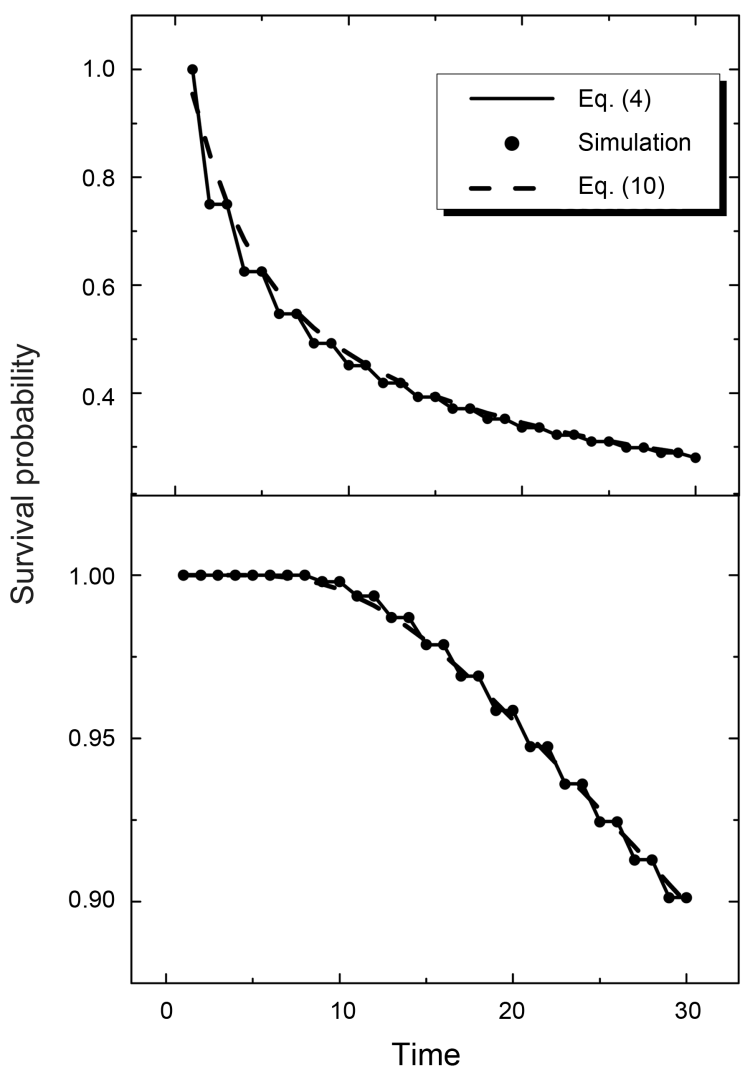

Figure 2. The time-dependence of the survival probability function for $x_{0}=2$ (upper) and 9 (lower). 
probability with the Smoluchowski boundary condition ${ }^{8}$

$$
S_{S M}\left(t ; x_{0}\right)=\operatorname{erf}\left(\frac{x_{0}}{\sqrt{4 D t}}\right)
$$

The equations (2), (3), and (4) is more fundamental functions than Eqs. (8), (9), and (11), respectively in that we can obtain Eq. (11) from Eq. (4), but Eq. (4) cannot be obtained from Eq. (11).

\section{Monte Carlo Simulation Results}

We can confirm the present results by using the Monte Carlo simulations. The simulations are performed by the latticed-based random walk model. ${ }^{2-6}$ A particle is initially implanted at $x_{0}$ and it starts moving in random directions. If the particle moves to the origin where the trap exists, the reaction occurs. Ten million ensembles are averaged to obtain the numerical results. In Figure 2, we plot the survival probabilities for $x_{0}=2$ and 9. One can see that Eq. (4) correctly reproduces the simulation results. Actually, Eq. (4) is much more efficient method than the simulation since it has no statistical noise. The difference between the results of Eqs. (4) and (10) is found to decrease as the time goes by.

In summary, we have reported the fundamental distribution functions for the lattice-based random walk model in one dimension for the nonreactive and the Smoluchowski boundary conditions. From the probability function, we obtain the discrete version of the survival probability for the Smoluchowski boundary condition. We confirm that these functions reduce to their well-known continuum version results. Since the lattice-based Monte Carlo simulations have been used for a variety of interesting chemical or biological systems, the present fundamental functions are of great use for these applications.

Acknowledgments. This work was supported by research funds from Dong-A University.

\section{References}

1. ben-Avraham, D.; Havlin, S. Diffusion and Reactions in Fractals and Disordered Systems; Cambridge University Press: Cambridge, 2000.

2. Kim, H.; Shin, S.; Lee, S.; Shin, K. J. J. Chem. Phys. 1996, 105, 7705.

3. Kim, H.; Shin, S.; Shin, K. J. J. Chem. Phys. 1998, 108, 5861.

4. Kim, H.; Shin, S.; Shin, K. J. Chem. Phys. Lett. 1998, 291, 341.

5. Kim, H.; Shin, K. J. Phys. Rev. E 2000, 61, 3426.

6. Kim, H. Chem. Phys. Lett. 2010, 484, 358.

7. Montroll, E. W.; Weiss, G. H. J. Math. Phys. 1965, 6, 167.

8. Carslaw, H. S.; Jaeger, J. C. Conduction of Heat in Solids, 2nd ed.; Oxford University Press: New York, 1986.

9. Feller, W. An Introduction to Probability Theory and Its Applications, 3rd ed.; Wiley: New York, 1968; Vol. 1. 